\title{
Some Properties of Certain Integral Operators on New Subclasses of Analytic Functions with Complex Order
}

\section{Aabed Mohammed and Maslina Darus}

School of Mathematical Sciences, Faculty of Science and Technology, Universiti Kebangsaan Malaysia, 43600 Bangi, Selangor, Malaysia

Correspondence should be addressed to Maslina Darus, maslina@ukm.my

Received 15 July 2012; Accepted 15 September 2012

Academic Editor: Roberto Natalini

Copyright (C 2012 A. Mohammed and M. Darus. This is an open access article distributed under the Creative Commons Attribution License, which permits unrestricted use, distribution, and reproduction in any medium, provided the original work is properly cited.

We define new subclasses of $p$-valent meromorphic functions with complex order. We prove some properties for certain integral operators on these subclasses.

\section{Introduction}

Let $\mathbb{U}=\{z \in \mathbb{C}:|z|<1\}$ be the open unit disc in the complex plane $\mathbb{C}, \mathbb{U}^{*}=\mathbb{U} \backslash\{0\}$, the punctured open unit disk. Let $\Sigma_{p}$ denote the class of meromorphic functions of the form

$$
f(z)=\frac{1}{z^{p}}+\sum_{n=0}^{\infty} a_{n} z^{n}, \quad(p \in \mathbb{N})
$$

which are analytic and $p$-valent in $\mathbb{U}^{*}$.

For $p=1$, we obtain the class of meromorphic functions $\Sigma$.

We say that a function $f \in \Sigma_{p}$ is the meromorphic $p$-valent starlike of order $\alpha(0 \leq \alpha<$ $p$ ) and belongs to the class $f \in \Sigma_{p}^{\star}(\alpha)$, if it satisfies the inequality

$$
-\mathfrak{R}\left(\frac{z f^{\prime}(z)}{f(z)}\right)>\alpha
$$


A function $f \in \Sigma_{p}$ is the meromorphic $p$-valent convex function of order $\alpha(0 \leq \alpha<p)$, if $f$ satisfies the following inequality:

$$
-\Re\left(1+\frac{z f^{\prime \prime}(z)}{f^{\prime}(z)}\right)>\alpha,
$$

and we denote this class by $\Sigma K_{p}(\alpha)$.

Many important properties and characteristics of various interesting subclasses of the class $\Sigma_{p}$ of meromorphically $p$-valent functions were investigated extensively by (among others) Uralegaddi and Somanatha [1, 2], Liu and Srivastava [3, 4], Mogra [5, 6], Srivastava et a1. [7], Aouf et al. [8, 9], Joshi and Srivastava [10], Owa et a1. [11], and Kulkarni et al. [12]. Now, for $f \in \Sigma_{p}$, we define the following new subclasses.

Definition 1.1. Let a function $f \in \Sigma_{p}$ be analytic in $\mathbb{U}^{*}$. Then $f$ is in the class $\Sigma_{p, \mathcal{M}}(\beta, b)$ if it satisfies the inequality

$$
\mathfrak{R}\left\{p-\frac{1}{b}\left(\frac{z f^{\prime}(z)}{f(z)}+p\right)\right\}<\beta,
$$

where

$$
p \in \mathbb{N}, \quad b \in \mathbb{C} \backslash\{0\}, \quad \beta>p .
$$

Definition 1.2. Let a function $f \in \Sigma_{p}$ be analytic in $\mathbb{U}^{*}$. Then $f$ is in the class $\Sigma_{p \mathcal{N}}(\beta, b)$ if it satisfies the inequality

$$
\mathfrak{R}\left\{p-\frac{1}{b}\left(\frac{z f^{\prime \prime}(z)}{f^{\prime}(z)}+p+1\right)\right\}<\beta
$$

where

$$
p \in \mathbb{N}, \quad b \in \mathbb{C} \backslash\{0\}, \quad \beta>p .
$$

Definition 1.3. Let a function $f \in \Sigma_{p}$ be analytic in $\mathbb{U}^{*}$. Then $f$ is in the class $\Sigma F_{p}(\beta, b)$ if it satisfies the inequality

$$
\mathfrak{R}\left\{p-\frac{1}{b}\left(\frac{z\left(z f^{\prime \prime}(z)+(p+2) f^{\prime}(z)\right)}{z f^{\prime}(z)+(p+1) f(z)}+p\right)\right\}<\beta,
$$

where

$$
p \in \mathbb{N}, \quad b \in \mathbb{C} \backslash\{0\}, \quad \beta>p .
$$

For $p=1$ in Definitions 1.1, 1.2, and 1.3, we obtain the following new subclasses of meromorphic functions $\Sigma$. 
Definition 1.4. Let a function $f \in \Sigma$ be analytic in $\mathbb{U}^{*}$. Then $f$ is in the class $\Sigma_{\mathscr{M}}(\beta, b)$ if it satisfies the inequality

$$
\mathfrak{R}\left\{1-\frac{1}{b}\left(\frac{z f^{\prime}(z)}{f(z)}+1\right)\right\}<\beta,
$$

where

$$
b \in \mathbb{C} \backslash\{0\}, \quad \beta>1 .
$$

Definition 1.5. Let a function $f \in \Sigma$ be analytic in $\mathbb{U}^{*}$. Then $f$ is in the class $\Sigma_{\mathcal{N}}(\beta, b)$ if it satisfies the inequality

$$
\mathfrak{R}\left\{1-\frac{1}{b}\left(\frac{z f^{\prime \prime}(z)}{f^{\prime}(z)}+2\right)\right\}<\beta,
$$

where

$$
b \in \mathbb{C} \backslash\{0\}, \quad \beta>1 .
$$

For $b=1$ in Definition 1.5, we obtain $\Sigma_{N}(\beta)$, the class of meromorphic function, introduced and studied by Wang et al. [13-15] (see [16-18]).

Definition 1.6. Let a function $f \in \Sigma$ be analytic in $\mathbb{U}^{*}$. Then $f$ is in the class $\Sigma F(\beta, b)$ if it satisfies the inequality

$$
\mathfrak{R}\left\{1-\frac{1}{b}\left(\frac{z\left(z f^{\prime \prime}(z)+3 f^{\prime}(z)\right)}{z f^{\prime}(z)+2 f(z)}+1\right)\right\}<\beta,
$$

where

$$
b \in \mathbb{C} \backslash\{0\}, \quad \beta>1 .
$$

Most recently, Mohammed and Darus [19] introduced the following two general integral operators of $p$-valent meromorphic functions $\Sigma_{p}$ :

$$
\begin{gathered}
\mathcal{F}_{p, \gamma_{1}, \ldots, \gamma_{n}}(z)=\frac{1}{z^{p+1}} \int_{0}^{z}\left(u^{p} f_{1}(u)\right)^{\gamma_{1}} \cdots\left(u^{p} f_{n}(u)\right)^{\gamma_{n}} d u \\
\partial_{p, \gamma_{1}, \ldots, \gamma_{n}}(z)=\frac{1}{z^{p+1}} \int_{0}^{z}\left(\frac{-u^{p+1}}{p} f_{1}^{\prime}(u)\right)^{\gamma_{1}} \cdots\left(\frac{-u^{p+1}}{p} f_{n}^{\prime}(u)\right)^{\gamma_{n}} d u
\end{gathered}
$$


where

$$
n, p \in \mathbb{N}, \quad j \in\{1,2,3, \ldots, n\}, \quad r_{j}>0
$$

For $p=1$ in (1.16) and (1.17), respectively, we obtain the general integral operators $\mathcal{F}_{1, \gamma_{1}, \ldots, \gamma_{n}}(z)=\mathscr{H}(z)$ and $\mathcal{2}_{1, \gamma_{1}, \ldots, \gamma_{n}}(z)=\mathscr{H}_{\gamma_{1}, \ldots, \gamma_{n}}(z)$, introduced by the authors in $[16,20]$.

\section{Main Results}

In this section, considering the above new subclasses we obtain for the integral operators $F_{1, \gamma_{1}, \ldots, \gamma_{n}}(z)$ and $2_{1, \gamma_{1}, \ldots, \gamma_{n}}(z)$ some sufficient conditions for a family of functions $f_{i}$ to be in the the above new subclasses.

Theorem 2.1. Let $f_{j} \in \Sigma_{p}$. If $f_{j} \in \Sigma_{p \mathcal{M}}\left(\beta_{j}, b\right)$, then

$$
\mathcal{F}_{p, \gamma_{1}, \ldots, \gamma_{n}}(z) \in \Sigma F_{p}(\mu, b)
$$

where

$$
\begin{gathered}
n, p \in \mathbb{N}, \quad j \in\{1, \ldots, n\}, \quad \gamma_{j}>0, \quad b \in \mathbb{C} \backslash\{0\}, \quad \beta_{j}>p, \\
\mu=p+\sum_{j=1}^{n} \gamma_{j}\left(\beta_{j}-p\right) .
\end{gathered}
$$

Proof. A differentiation of $\mathcal{F}_{p, \gamma_{1}, \ldots, \gamma_{n}}(z)$ which is defined in (1.16), we get

$$
\begin{gathered}
z^{p+1} \mathcal{F}_{p, \gamma_{1}, \ldots, \gamma_{n}}^{\prime}(z)+(p+1) z^{p} \mathcal{F}_{p, \gamma_{1}, \ldots, \gamma_{n}}(z)=\left(z^{p} f_{1}(z)\right)^{\gamma_{1}} \cdots\left(z^{p} f_{n}(z)\right)^{\gamma_{n}}, \\
z^{p+1} \mathcal{F}_{p, \gamma_{1}, \ldots, \gamma_{n}}^{\prime \prime}(z)+2(p+1) z^{p} \mathcal{F}_{p, \gamma_{1}, \ldots, \gamma_{n}}^{\prime}(z)+p(p+1) z^{p-1} \mathcal{F}_{p, \gamma_{1}, \ldots, \gamma_{n}}(z) \\
=\sum_{j=1}^{n} \gamma_{j}\left(\frac{z^{p} f_{j}^{\prime}(z)+p z^{p-1} f_{j}(z)}{z^{p} f_{j}(z)}\right)\left[\left(z^{p} f_{1}(z)\right)^{\gamma_{1}} \cdots\left(z^{p} f_{n}(z)\right)^{\gamma_{n}}\right],
\end{gathered}
$$

Then from (2.3), we obtain

$$
\begin{aligned}
& \frac{z^{p+1} \mathcal{F}_{p, \gamma_{1}, \ldots, \gamma_{n}}^{\prime \prime}(z)+2(p+1) z^{p} \mathcal{F}_{p, \gamma_{1}, \ldots, \gamma_{n}}^{\prime}(z)+p(p+1) z^{p-1} \mathcal{F}_{p, \gamma_{1}, \ldots, \gamma_{n}}(z)}{z^{p+1} \mathcal{F}_{p, \gamma_{1}, \ldots, \gamma_{n}}^{\prime}(z)+(p+1) z^{p} \mathcal{F}_{p, \gamma_{1}, \ldots, \gamma_{n}}(z)} \\
& \quad=\sum_{j=1}^{n} \gamma_{j}\left(\frac{f_{j}^{\prime}(z)}{f_{j}(z)}+\frac{p}{z}\right) .
\end{aligned}
$$


By multiplying (2.4) with $z$ yield,

$$
\begin{aligned}
& \frac{z^{p+1} \mathscr{F}_{p, \gamma_{1}, \ldots, \gamma_{n}}^{\prime \prime}(z)+2(p+1) z^{p} \mathcal{F}_{p, \gamma_{1}, \ldots, \gamma_{n}}^{\prime}(z)+p(p+1) z^{p-1} \mathcal{F}_{p, \gamma_{1}, \ldots, \gamma_{n}}(z)}{z^{p} \mathcal{F}_{p, \gamma_{1}, \ldots, \gamma_{n}}^{\prime}(z)+(p+1) z^{p-1} \mathscr{F}_{p, \gamma_{1}, \ldots, \gamma_{n}}(z)} \\
& \quad=\sum_{j=1}^{n} \gamma_{j}\left(\frac{z f_{j}^{\prime}(z)}{f_{j}(z)}+p\right) .
\end{aligned}
$$

That is equivalent to

$$
\begin{aligned}
& \frac{z^{p+1} \mathcal{F}_{p, \gamma_{1}, \ldots, \gamma_{n}}^{\prime \prime}(z)+(p+2) z^{p} \mathcal{F}_{p, \gamma_{1}, \ldots, \gamma_{n}}^{\prime}(z)}{z^{p} \mathcal{F}_{p, \gamma_{1}, \ldots, \gamma_{n}}^{\prime}(z)+(p+1) z^{p-1} \mathcal{F}_{p, \gamma_{1}, \ldots, \gamma_{n}}(z)}+p=\sum_{j=1}^{n} \gamma_{j}\left(\frac{z f_{j}^{\prime}(z)}{f_{j}(z)}+p\right) ; \\
& \frac{z\left(z \mathcal{F}_{p, \gamma_{1}, \ldots, \gamma_{n}}^{\prime \prime}(z)+(p+2) \mathcal{F}_{p, \gamma_{1}, \ldots, \gamma_{n}}^{\prime}(z)\right)}{z \mathcal{F}_{p, \gamma_{1}, \ldots, \gamma_{n}}^{\prime}(z)+(p+1) \mathcal{F}_{p, \gamma_{1}, \ldots, \gamma_{n}}(z)}+p=\sum_{j=1}^{n} \gamma_{j}\left(\frac{z f_{j}^{\prime}(z)}{f_{j}(z)}+p\right) .
\end{aligned}
$$

Equivalently, the above can be written as

$$
\begin{array}{r}
p-\frac{1}{b}\left(\frac{z\left(z \mathcal{F}_{p, \gamma_{1}, \ldots, \gamma_{n}}^{\prime \prime}(z)+(p+2) \mathscr{F}_{p, \gamma_{1}, \ldots, \gamma_{n}}^{\prime}(z)\right)}{z \mathcal{F}_{p, \gamma_{1}, \ldots, \gamma_{n}}^{\prime}(z)+(p+1) \mathcal{F}_{p, \gamma_{1}, \ldots, \gamma_{n}}(z)}+p\right) \\
\quad=\sum_{j=1}^{n} r_{j}\left\{p-\frac{1}{b}\left(\frac{z f_{j}^{\prime}(z)}{f_{j}(z)}+p\right)\right\}+p-p \sum_{j=1}^{n} r_{j} .
\end{array}
$$

Taking the real part of both terms of (2.7), we have

$$
\begin{aligned}
\mathfrak{R}\{p- & \left.\frac{1}{b}\left(\frac{z\left(z \mathcal{F}_{p, \gamma_{1}, \ldots, \gamma_{n}}^{\prime \prime}(z)+(p+2) \mathcal{F}_{p, \gamma_{1}, \ldots, \gamma_{n}}^{\prime}(z)\right)}{z \mathcal{F}_{p, \gamma_{1}, \ldots, \gamma_{n}}^{\prime}(z)+(p+1) \mathcal{F}_{p, \gamma_{1}, \ldots, \gamma_{n}}(z)}+p\right)\right\} \\
= & \sum_{j=1}^{n} r_{j} \Re\left\{p-\frac{1}{b}\left(\frac{z f_{j}^{\prime}(z)}{f_{j}(z)}+p\right)\right\}+p-p \sum_{j=1}^{n} \gamma_{j} .
\end{aligned}
$$

Sine $f_{j} \in \Sigma_{p \mathcal{M}}\left(\beta_{j}, b\right)$, we get

$$
\mathfrak{R}\left\{p-\frac{1}{b}\left(\frac{z\left(z \mathcal{F}_{p, \gamma_{1}, \ldots, \gamma_{n}}^{\prime \prime}(z)+(p+2) \mathcal{F}_{p, \gamma_{1}, \ldots, \gamma_{n}}^{\prime}(z)\right)}{z \mathcal{F}_{p, \gamma_{1}, \ldots, \gamma_{n}}^{\prime}(z)+(p+1) \mathcal{F}_{p, \gamma_{1}, \ldots, \gamma_{n}}(z)}+p\right)\right\}<\sum_{j=1}^{n} \gamma_{j} \beta_{j}+p-p \sum_{j=1}^{n} \gamma_{j}
$$


That is,

$$
\mathfrak{R}\left\{p-\frac{1}{b}\left(\frac{z\left(z \mathcal{F}_{p, \gamma_{1}, \ldots, \gamma_{n}}^{\prime \prime}(z)+(p+2) \mathcal{F}_{p, \gamma_{1}, \ldots, \gamma_{n}}^{\prime}(z)\right)}{z \mathcal{F}_{p, \gamma_{1}, \ldots, \gamma_{n}}^{\prime}(z)+(p+1) \mathcal{F}_{p, \gamma_{1}, \ldots, \gamma_{n}}(z)}+p\right)\right\}<p+\sum_{j=1}^{n} \gamma_{j}\left(\beta_{j}-p\right)
$$

Then

$$
\mathcal{F}_{p, \gamma_{1}, \ldots, \gamma_{n}}(z) \in \Sigma F_{p}(\mu, b), \quad \mu=p+\sum_{j=1}^{n} \gamma_{j}\left(\beta_{j}-p\right)
$$

This completes the proof.

Theorem 2.2. Let $f_{j} \in \Sigma_{p}$. If $f_{j} \in \Sigma_{p \mathcal{N}}\left(\beta_{j}, b\right)$, then

$$
\partial_{p, \gamma_{1}, \ldots, \gamma_{n}}(z) \in \Sigma F_{p}(\mu, b)
$$

where

$$
\begin{gathered}
n, p \in \mathbb{N}, \quad j \in\{1, \ldots, n\}, \quad \gamma_{j}>0, \quad b \in \mathbb{C} \backslash\{0\}, \quad \beta_{j}>p, \\
\mu=p+\sum_{j=1}^{n} \gamma_{j}\left(\beta_{j}-p\right) .
\end{gathered}
$$

Proof. A differentiation of $\partial_{p, \gamma_{1}, \ldots, \gamma_{n}}(z)$, which is defined in (1.17), we get

$$
\begin{aligned}
& z^{p+1} \partial_{p, \gamma_{1}, \ldots, \gamma_{n}}^{\prime}(z)+(p+1) z^{p} \partial_{p, \gamma_{1}, \ldots, \gamma_{n}}(z)=\left(\frac{-z^{p+1}}{p} f_{1}^{\prime}(z)\right)^{\gamma_{1}} \cdots\left(\frac{-z^{p+1}}{p} f_{n}^{\prime}(z)\right)^{\gamma_{n}}, \\
& z^{p+1} \partial_{p, \gamma_{1}, \ldots, \gamma_{n}}^{\prime \prime}(z)+2(p+1) z^{p} \partial_{p, \gamma_{1}, \ldots, \gamma_{n}}^{\prime}(z)+p(p+1) z^{p-1} \partial_{p, \gamma_{1}, \ldots, \gamma_{n}}(z) \\
& =\sum_{j=1}^{n} \gamma_{j}\left(\frac{z^{p+1} f_{j}^{\prime \prime}(z)+(p+1) z^{p} f_{j}^{\prime}(z)}{z^{p+1} f_{j}^{\prime}(z)}\right)\left[\left(\frac{-z^{p+1}}{p} f_{1}^{\prime}(z)\right)^{\gamma_{1}} \cdots\left(\frac{-z^{p+1}}{p} f_{n}^{\prime}(z)\right)^{\gamma_{n}}\right] .
\end{aligned}
$$

Then from (2.14), we obtain

$$
\begin{aligned}
& \frac{z^{p+1} \partial_{p, \gamma_{1}, \ldots, \gamma_{n}}^{\prime \prime}(z)+2(p+1) z^{p} \partial_{p, \gamma_{1}, \ldots, \gamma_{n}}^{\prime}(z)+p(p+1) z^{p-1} \partial_{p, \gamma_{1}, \ldots, \gamma_{n}}(z)}{z^{p} \partial_{p, \gamma_{1}, \ldots, \gamma_{n}}^{\prime}(z)+(p+1) z^{p-1} \partial_{p, \gamma_{1}, \ldots, \gamma_{n}}(z)} \\
& \quad=\sum_{j=1}^{n} \gamma_{j}\left(\frac{z f_{j}^{\prime \prime}(z)}{f_{j}^{\prime}(z)}+p+1\right) .
\end{aligned}
$$


Journal of Applied Mathematics

That is equivalent to

$$
\frac{z\left(z \partial_{p, \gamma_{1}, \ldots, \gamma_{n}}^{\prime \prime}(z)+(p+2) \partial_{p, \gamma_{1}, \ldots, \gamma_{n}}^{\prime}(z)\right)}{z \partial_{p, \gamma_{1}, \ldots, \gamma_{n}}^{\prime}(z)+(p+1) \partial_{p, \gamma_{1}, \ldots, \gamma_{n}}(z)}+p=\sum_{j=1}^{n} \gamma_{j}\left(\frac{z f_{j}^{\prime \prime}(z)}{f_{j}^{\prime}(z)}+p+1\right) .
$$

Equivalently, the above can be written as

$$
\begin{aligned}
p & -\frac{1}{b}\left(\frac{z\left(z \partial_{p, \gamma_{1}, \ldots, \gamma_{n}}^{\prime \prime}(z)+(p+2) \partial_{p, \gamma_{1}, \ldots, \gamma_{n}}^{\prime}(z)\right)}{z \partial_{p, \gamma_{1}, \ldots, \gamma_{n}}^{\prime}(z)+(p+1) \partial_{p, \gamma_{1}, \ldots, \gamma_{n}}(z)}+p\right) \\
& =\sum_{j=1}^{n} \gamma_{j}\left\{p-\frac{1}{b}\left(\frac{z f_{j}^{\prime \prime}(z)}{f_{j}^{\prime}(z)}+p+1\right)\right\}+p-p \sum_{j=1}^{n} \gamma_{j} .
\end{aligned}
$$

Taking the real part of both terms of (2.17), we have

$$
\begin{gathered}
\mathfrak{R}\left\{p-\frac{1}{b}\left(\frac{z\left(z \partial_{p, \gamma_{1}, \ldots, \gamma_{n}}^{\prime \prime}(z)+(p+2) \partial_{p, \gamma_{1}, \ldots, \gamma_{n}}^{\prime}(z)\right)}{z \partial_{p, \gamma_{1}, \ldots, \gamma_{n}}^{\prime}(z)+(p+1) \partial_{p, \gamma_{1}, \ldots, \gamma_{n}}(z)}+p\right)\right\} \\
=\sum_{j=1}^{n} \gamma_{j} \mathfrak{R}\left\{p-\frac{1}{b}\left(\frac{z f_{j}^{\prime \prime}(z)}{f_{j}^{\prime}(z)}+p+1\right)\right\}+p-p \sum_{j=1}^{n} \gamma_{j} .
\end{gathered}
$$

Sine $f_{j} \in \Sigma_{p \mathcal{N}}\left(\beta_{j}, b\right)$, we get

$$
\mathfrak{R}\left\{p-\frac{1}{b}\left(\frac{z\left(z \partial_{p, \gamma_{1}, \ldots, \gamma_{n}}^{\prime \prime}(z)+(p+2) \partial_{p, \gamma_{1}, \ldots, \gamma_{n}}^{\prime}(z)\right)}{z \partial_{p, \gamma_{1}, \ldots, \gamma_{n}}^{\prime}(z)+(p+1) \partial_{p, \gamma_{1}, \ldots, \gamma_{n}}(z)}+p\right)\right\}<\sum_{j=1}^{n} \gamma_{j} \beta_{j}+p-p \sum_{j=1}^{n} \gamma_{j} .
$$

That is,

$$
\mathfrak{R}\left\{p-\frac{1}{b}\left(\frac{z\left(z \partial_{p, \gamma_{1}, \ldots, \gamma_{n}}^{\prime \prime}(z)+(p+2) \partial_{p, \gamma_{1}, \ldots, \gamma_{n}}^{\prime}(z)\right)}{z \partial_{p, \gamma_{1}, \ldots, \gamma_{n}}^{\prime}(z)+(p+1) \partial_{p, \gamma_{1}, \ldots, \gamma_{n}}(z)}+p\right)\right\}<p+\sum_{j=1}^{n} \gamma_{j}\left(\beta_{j}-p\right) .
$$

Then

$$
\partial_{p, \gamma_{1}, \ldots, \gamma_{n}}(z) \in \Sigma F_{p}(\mu, b), \quad \mu=p+\sum_{j=1}^{n} \gamma_{j}\left(\beta_{j}-p\right) .
$$

This completes the proof.

Putting $p=1$ in Theorem 2.1, we have the following. 
Theorem 2.3. Let $f_{j} \in \Sigma$. If $f_{j} \in \Sigma_{\mathcal{M}}\left(\beta_{j}, b\right)$, then

$$
\mathscr{H}_{n}(z) \in \Sigma F(\mu, b),
$$

where

$$
\begin{gathered}
n \in \mathbb{N}, \quad j \in\{1, \ldots, n\}, \quad \gamma_{j}>0, \quad b \in \mathbb{C} \backslash\{0\}, \quad \beta_{j}>1, \\
\mu=1+\sum_{j=1}^{n} \gamma_{j}\left(\beta_{j}-1\right) .
\end{gathered}
$$

Putting $p=1$ in Theorem 2.2, we have the following.

Theorem 2.4. Let $f_{j} \in \Sigma$. If $f_{j} \in \Sigma_{\mathcal{N}}\left(\beta_{j}, b\right)$, then

$$
\mathscr{H}_{\gamma_{1}, \ldots, \gamma_{n}}(z) \in \Sigma F(\mu, b)
$$

where

$$
\begin{gathered}
n \in \mathbb{N}, \quad j \in\{1, \ldots, n\}, \quad \gamma_{j}>0, \quad b \in \mathbb{C} \backslash\{0\}, \quad \beta_{j}>1, \\
\mu=1+\sum_{j=1}^{n} \gamma_{j}\left(\beta_{j}-1\right) .
\end{gathered}
$$

for other work that we can look at regarding integral operators see [17, 21, 22].

\section{Acknowledgment}

This work was supported by UKM-ST-06-FRGS0244-2010 and LRGS/TD/2011/UKM/ $\mathrm{ICT} / 03 / 02$.

\section{References}

[1] B. A. Uralegaddi and C. Somanatha, "New criteria for meromorphic starlike univalent functions," Bulletin of the Australian Mathematical Society, vol. 43, no. 1, pp. 137-140, 1991.

[2] B. A. Uralegaddi and C. Somanatha, "Certain classes of meromorphic multivalent functions," Tamkang Journal of Mathematics, vol. 23, no. 3, pp. 223-231, 1992.

[3] J.-L. Liu and H. M. Srivastava, "A linear operator and associated families of meromorphically multivalent functions," Journal of Mathematical Analysis and Applications, vol. 259, no. 2, pp. 566-581, 2001.

[4] J.-L. Liu and H. M. Srivastava, "Some convolution conditions for starlikeness and convexity of meromorphically multivalent functions," Applied Mathematics Letters, vol. 16, no. 1, pp. 13-16, 2003.

[5] M. L. Mogra, "Meromorphic multivalent functions with positive coefficients. I," Mathematica Japonica, vol. 35, no. 1, pp. 1-11, 1990.

[6] M. L. Mogra, "Meromorphic multivalent functions with positive coefficients. II," Mathematica Japonica, vol. 35, no. 6, pp. 1089-1098, 1990.

[7] H. M. Srivastava, H. M. Hossen, and M. K. Aouf, "A unified presentation of some classes of meromorphically multivalent functions," Computers \& Mathematics with Applications, vol. 38, no. 11-12, pp. 63-70, 1999. 
[8] M. K. Aouf and H. M. Hossen, “New criteria for meromorphic p-valent starlike functions," Tsukuba Journal of Mathematics, vol. 17, no. 2, pp. 481-486, 1993.

[9] M. K. Aouf and M. H. Srivastava, "Anew criterion for meromorphically $p$-valent convex functions of order alpha," Mathematical Sciences Research Hot-Line, vol. 1, no. 8, pp. 7-12, 1997.

[10] S. B. Joshi and H. M. Srivastava, "A certain family of meromorphically multivalent functions," Computers $\mathcal{E}$ Mathematics with Applications, vol. 38, no. 3-4, pp. 201-211, 1999.

[11] S. Owa, H. E. Darwish, and M. K. Aouf, "Meromorphic multivalent functions with positive and fixed second coefficients," Mathematica Japonica, vol. 46, no. 2, pp. 231-236, 1997.

[12] S. R. Kulkarni, U. H. Naik, and H. M. Srivastava, "A certain class of meromorphically p-valent quasiconvex functions," Panamerican Mathematical Journal, vol. 8, no. 1, pp. 57-64, 1998.

[13] Z.-G. Wang, Z.-H. Liu, and R.-G. Xiang, "Some criteria for meromorphic multivalent starlike functions," Applied Mathematics and Computation, vol. 218, no. 3, pp. 1107-1111, 2011.

[14] Z.-G. Wang, Y. Sun, and Z.-H. Zhang, "Certain classes of meromorphic multivalent functions," Computers $\mathcal{E}$ Mathematics with Applications, vol. 58, no. 7, pp. 1408-1417, 2009.

[15] Z.-G. Wang, Z.-H. Liu, and A. Cătaş, “On neighborhoods and partial sums of certain meromorphic multivalent functions," Applied Mathematics Letters, vol. 24, no. 6, pp. 864-868, 2011.

[16] A. Mohammed and M. Darus, "A new integral operator for meromorphic functions," Acta Universitatis Apulensis, no. 24, pp. 231-238, 2010.

[17] A. Mohammed and M. Darus, "Integral operators on new families of meromorphic functions of complex order," Journal of Inequalities and Applications, vol. 2011, article 121, 12 pages, 2011.

[18] B. A. Frasin, "On an integral operator of meromorphic functions," Matematichki Vesnik, vol. 64, no. 2, pp. 167-172, 2012.

[19] A. Mohammed and M. Darus, "The order of starlikeness of new $p$-valent meromorphic functions," International Journal of Mathematical Analysis, vol. 6, no. 27, pp. 1329-1340, 2012.

[20] A. Mohammed and M. Darus, "Starlikeness properties of a new integral operator for meromorphic functions," Journal of Applied Mathematics, vol. 2011, Article ID 804150, 8 pages, 2011.

[21] A. Mohammed and M. Darus, "New properties for certain integral operators," International Journal of Mathematical Analysis, vol. 4, no. 41-44, pp. 2101-2109, 2010.

[22] N. Breaz, D. Breaz, and M. Darus, "Convexity properties for some general integral operators on uniformly analytic functions classes," Computers $\mathcal{E}$ Mathematics with Applications, vol. 60, no. 12, pp. 3105-3107, 2010. 


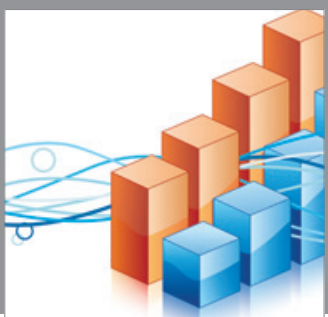

Advances in

Operations Research

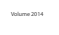

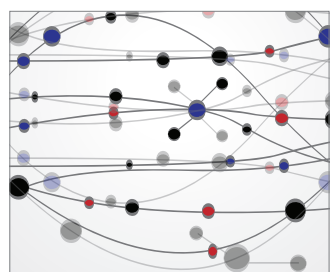

\section{The Scientific} World Journal
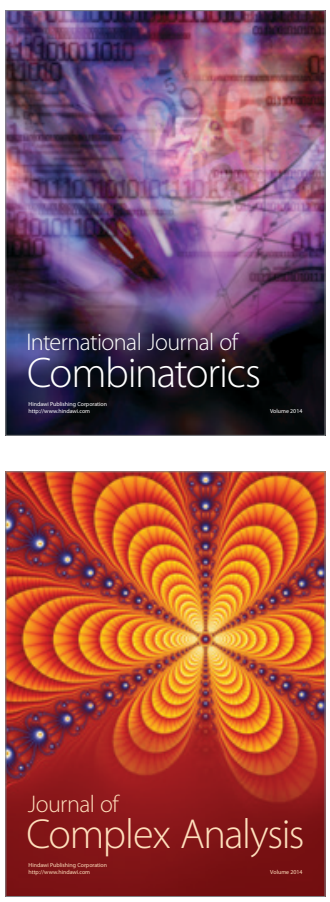

International Journal of

Mathematics and

Mathematical

Sciences
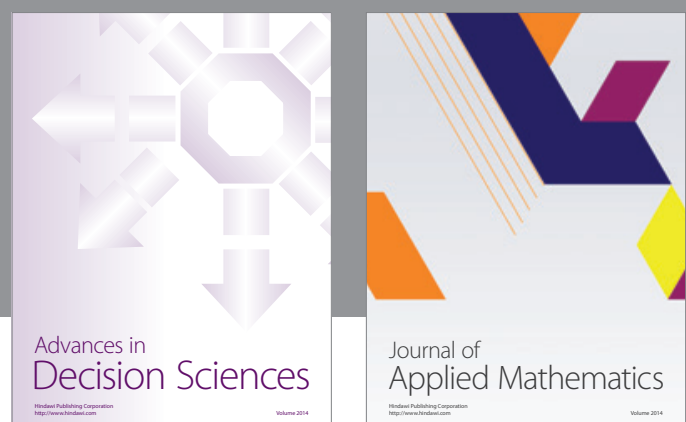

Journal of

Applied Mathematics
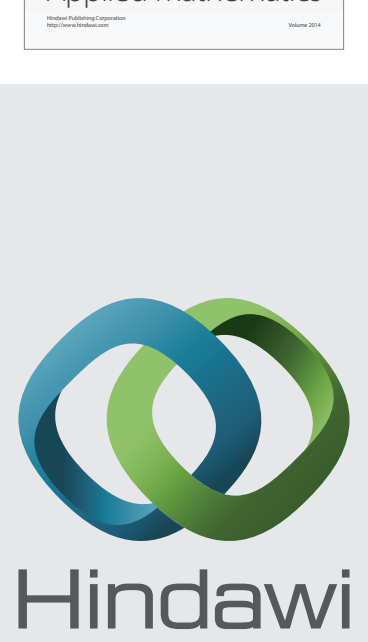

Submit your manuscripts at http://www.hindawi.com
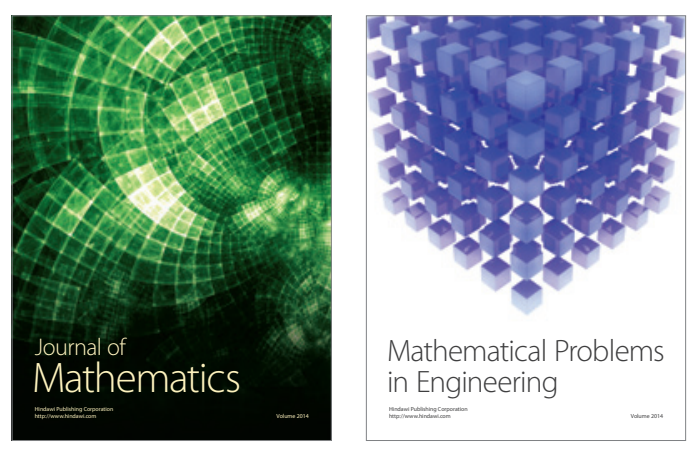

Mathematical Problems in Engineering
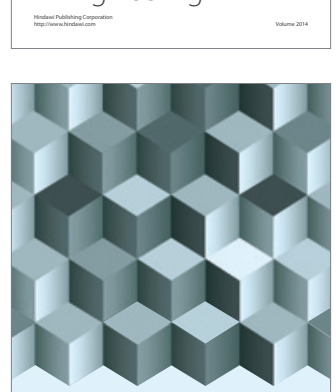

Journal of

Function Spaces
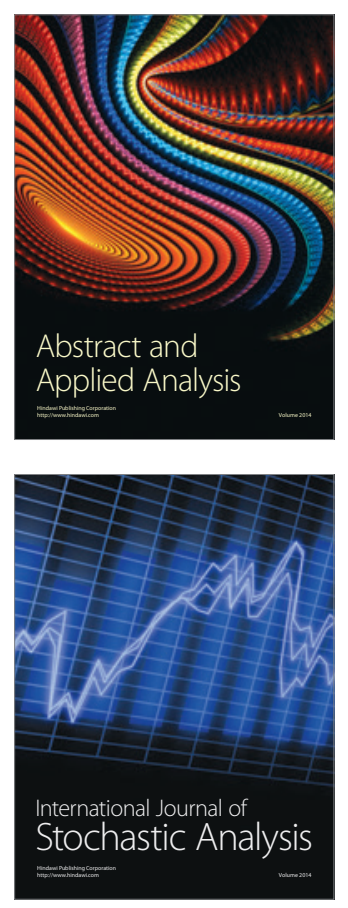

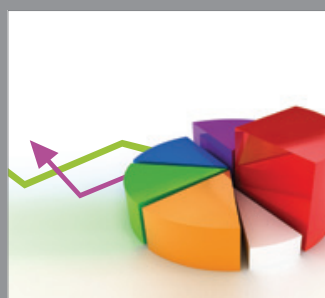

ournal of

Probability and Statistics

Promensencen
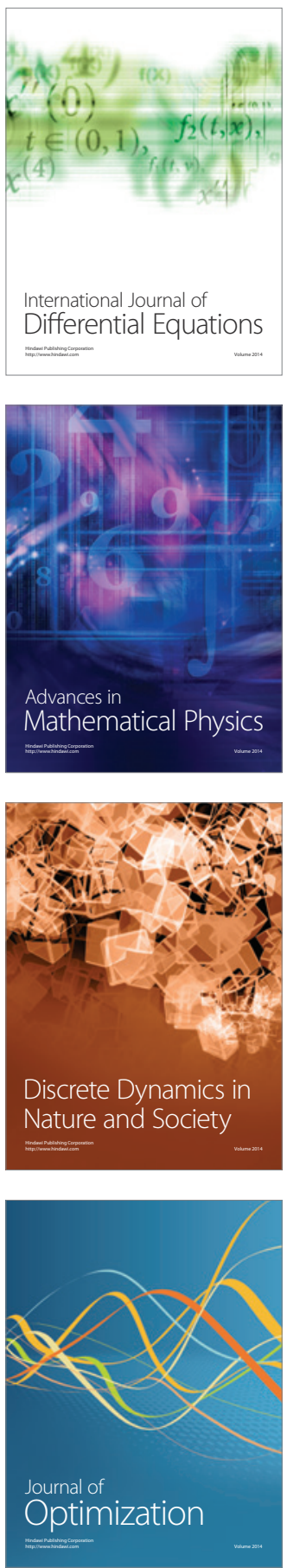